\title{
AC 2011-2247: INCREASING STEM ACCESSIBILITY FOR STUDENTS WITH COGNITIVE DISABILITIES VIA INTERACTIVE CURRICULUM
}

\section{Ethan E Danahy, Tufts University}

Ethan Danahy received the B.S. and M.S. degrees in Computer Science in 2000 and 2002, respectively, and a Ph.D. degree in Electrical Engineering in 2007, all at Tufts University, Medford, MA. Within the School of Engineering at Tufts University, he is currently a Research Assistant Professor in the Department of Computer Science. Additionally, he acts as the Engineering Research Program Director at the Center for Engineering Education and Outreach (CEEO), where he manages educational technology development projects while researching innovative and interactive techniques for assisting teachers with performing engineering education and communicating robotics concepts to students spanning the K-12 through university age range.

Ashley Russell, CEEO 


\title{
Improving STEM Learning through Accessible RoboBooks
}

\begin{abstract}
In this study the researchers conducted an inclusive and iterative design protocol in order to develop an accessible, interactive engineering curriculum delivery tool and promote equitable learning opportunities for students with learning and cognitive disabilities. The software interface is utilized in this research to promote teaching science through engineering by leveraging features inherent to the platform. Three interface design iterations were tested in the classroom for this pilot study, with findings from each iteration directing improvements for the subsequent design phase. Outcomes were derived from student navigation tracking, questionnaires, focus groups, and teacher interviews. By approaching the research from an inclusive design perspective through solicitation of teacher and student input, this research aims to improve the interaction between students and software and ultimately promote gains in student learning in STEM education through improved accessibility to information and means of knowledge expression.
\end{abstract}

Introduction

Federal legislation aspires to improve educational outcomes for students with disabilities by instituting their right to participate in the general education curriculum, however national assessment data indicate significant performance differences between students with and without disabilities ${ }^{1}$. These changes in legislation have promoted an integrated student body, which endorses the need for tools that allow all learners access to information and promote a successful learning environment ${ }^{2}$. Yet, while these expectations for students with disabilities have increased considerably, the instructional materials used in the classroom generally remain limited to printed text and paper-andpencil activities ${ }^{3}$. These media continue to be the primary means for acquiring information and demonstrating knowledge in STEM education, which consequently pose barriers to scientific learning. Such fixed media creates an unnecessary challenge to students who might not only struggle with the content, but also struggle with reading, taking notes, organizing information and analyzing and presenting data. Moreover, the National Science Education Standards have shifted the emphasis from learning acquisition and teacher presentation to active student observation and interaction ${ }^{4}$. This only further exacerbates the barriers to STEM learning for students with disabilities, as it requires higher-level scientific reasoning and critical thinking. Using higher-order thinking skills with specific content knowledge in problem-solving activities can be challenging for all students, but especially for those with learning disabilities.

Educational technology is a tool that can accommodate varied learner needs and potentially close gaps in education performance for students with disabilities. Such digital platforms can provide supports and scaffolds that would otherwise be limited by use of only fixed media for curriculum presentation. However, considerations must be made in the design, evaluation, and implementation of educational technology so that as a tool it is usable, accessible, appropriate, and promotes gains for students as well as the 
larger educational system.

The Need

Learners using software applications leverage different skills and require wide range of motivations and objectives from the technology ${ }^{5}$. Consequently, different approaches for designing technology are needed in order to support users in achieving learning requirements. To be well designed, these software environments should enable students to learn by doing, receive immediate feedback, continually refine understanding, and create new knowledge structures ${ }^{6}$. This role for educational technology extends beyond typical user needs, but must also aid in the learning process through information acquisition, processing, and knowledge expression. Furthermore, educational technology should "support adaptability and flexibility, to enable appropriate modes of learning, and to foster interactive involvement of the learner with the educational materials" 7 . Notably, these goals can be well supported by current technology trends. Yet, van Dam et al. ${ }^{7}$ maintains there is no one-size-fit-all solution for educational technology. Furthermore, without accommodating for diverse learning styles, software media can be as limiting as fixed media.

Extend the thought of accommodation for diverse learning styles to the current classroom, an environment that now includes an increasingly diverse range of learners and their associated needs. Students that struggle with a range of behavioral, cognitive, and learning impediments work alongside students with a range of learning styles. And with engineering projects, which promote more hands-on work, these students need more supports for self-directed study and staying on task when the teacher's attention might be elsewhere due to increased administration produced by these type of activities. In designing educational technology for all, universal accessibility is a pertinent and conscientious design criterion for the development of successful educational technology that can support the aforementioned role of technology in the classroom for all learners.

The needs of an integrated student body include accommodation of all learner types and consideration of a wide range in capacity for information acquisition, processing, and subsequent expression of understanding. However, the cognitively disabled population has been largely overlooked in the field of system design and improvements for universal accessibility and design practices for software development are, for the most part, omitted from leading human computer interaction research ${ }^{8}$. Accordingly, interface design methods for working with, and for, the cognitively disabled population are limited; design guidelines are often vague or directed towards specific populations. Design methods and principles to promote universal and learner-centered design would provide valuable information to software developers that could allow them to address the needs of the end users.

\section{Educational Objectives}

Universal design for learning (UDL) is a set of principles for designing curriculum that provides a flexible approach and equal opportunities for learning. The 
three guiding principles for UDL are to provide multiple ways of representing content, multiple methods of engagement, and multiple ways of expression ${ }^{9,2}$. For the design of educational technology, the integration of UDL principles promotes improved accessibility for learners.

In the UDL context, content presentation could be addressed by providing text in digital media, which is accompanied by audio, additional graphics or videos, and cognitive aids such as highlighting, hints, and concept maps. Engagement could be supported by the provision of intermittent feedback, integrated activities, simulations and games. Tools for expression could include text input, audio record, image capture, and movie record. UDL features can be utilized in several manners to best support the content and to aid in information processing and conceptual understanding. By making instructional goals, strategies, and materials flexible in these ways, potential barriers to learning are lowered and opportunities to learn are increased.

By integrating UDL tools into a digital platform designed for cultivating engineering education in $\mathrm{K}-12$, the researchers aim to provide a means for improving STEM learning outcomes for all students. The software interface is utilized in this research to promote teaching science through engineering by leveraging features inherent to the platform. Learning through design is reflected in the software interface (called RoboBooks, described in detail later) by navigation features that support exploration, opportunities for analyzing problem states, modeling of solutions, and reflection through a journaling process.

\section{Theoretical Framework}

Soloway, Guzdial, and Hay ${ }^{10}$ indicated the need for user interface design to be expanded to that of learner-centered design for the development of educational software. Learner-centered design adds several aspects to software design that include: selection of a pedagogical foundation \& appropriate technology, prototyping, formative evaluation (which includes both usability testing and the evaluation of learning outcomes), iterative design, and a final summative evaluation ${ }^{11}$. As this procedure follows design in research concepts, the researchers believe that adopting the learner centered design practices can inform the design of the software such that it is appropriate and can consequently provide a means for improved learning outcomes, greater adoption in the classroom, and promote gains to STEM education.

The research presented here will discuss the pedagogical foundation and technology and focus on the prototyping, the iterative design process, and usability testing as part of the initial formative evaluation of integrating UDL tools with educational technology. This paper will also discuss preliminary learning evaluation in context of goals for the final design phase. The full study, in-progress, will additionally include evaluation of learning outcomes and a final summative evaluation for the final, refined interface but will not be presented in this paper. 
The Approach

The primary goal of this research is to integrate UDL tools into the engineering educational technology, RoboBooks, and provide students with a usable, accessible interface. This educational platform allows interactive, constructivist, inquiry-based curriculum delivery, components of learning through design ${ }^{12,13}$. The UDL tools promote equitable access for all students to this platform. By adopting learner centered design theory, the integration of platform and tools to support the curriculum presentation will include teacher and student feedback to inform the design, ensure usability, and ultimately provide an opportunity for student achievement via multimedia learning. The researchers from Tufts University's Center for Engineering Education and Outreach (CEEO) have teamed with two public high schools in Boston and CAST (the Center for Applied Special Technology) to best develop UDL supports in RoboBooks, and address the needs of the students and teachers.

\section{Technology}

The RoboBooks software platform, designed and developed at the CEEO, is an interactive digital workbook. Learning, according to Piaget, involves the construction of new knowledge from existing knowledge through the manipulation of artifacts and direct observation of consequent behavior ${ }^{14}$. The constructivist theory concept has been extended by Papert to constructivism, the pedagogical theory from which RoboBooks was derived. As an interactive notebook, RoboBooks provides a manipulable interface, live connection to hardware, and interactivity with real-time data access, which is ideal for engineering related activities that require all these aspects ${ }^{12}$. Multimedia elements such as text, images, audio, and movies allow students to explore content in a variety of ways. RoboBooks supports students in scientific investigations through interactive presentation of material and dynamic tools for documentation and journaling of the students' work. As a learning environment, RoboBooks uses LEGO robotics tool sets, supporting students in conducting experiments to develop ideas and reflecting on the design process ${ }^{15}$. RoboBooks is currently being used in a wide range of classrooms, ranging from middle-school engineering projects surrounding NASA curriculum to university-level robotics engineering coursework ${ }^{12,15}$. Ultimately, the exploration, manipulation, and creation through the RoboBooks platform promote the construction and expression of knowledge schemata in complex problem-solving arenas.

The UDL RoboBook interface is being developed with high school students and teachers and provides curriculum presentation via text, audio, graphics, and multimedia. The software environment augments current learning techniques while extending overall opportunities by means of interactivity and real time feedback ${ }^{12}$. The interactive and selfdirected nature of this software tool provides scaffolding for student learning, which gives the teacher more opportunities to facilitate student learning rather than expend resources on classroom management, particularly true for classrooms engaged in engineering activities. 


\section{Prototype}

Functional user characteristics across learning disabilities can be categorized, interdependently, as: (1) reading (2) memory, (3) metacognitive, and (4) search and navigation $^{16}$. Gribbons ${ }^{8}$ proposes that each category encapsulates a broad list of deficiencies and many deficiencies are connected to others. Within the category of reading, for example, one draws on both memory and metacognitive skills. Likewise, search and navigation places load on working and long-term memory and needs monitoring from metacognitive functioning. Each category and interdependency suggests particular user requirements.

User requirements, in turn, lead to particular design guidelines. For example, using visual and auditory prompts provides an alternative strategy for users who may be confronted with an initial reading barrier ${ }^{8}$. Low information density is a complementary strategy for reading, as well as using illustrations to support text, emphasizing connections, and presenting content in sequence. Metacognitive support could be promoted by immediate communication of goals. Effective navigation and search design practices include the use of labels, identified paths, site maps, and use of redundant cues.

Using these initial user characteristic guidelines, an initial interface was constructed. This prototype was used to structure the information, ensure consistent navigation, and promote overall usability for the population demographic. The prototype was reviewed with the teachers prior to implementing the first phase design.

\section{Iterative Design}

For initial classroom testing, a three-phase design plan was created to establish objectives, conduct preliminary usability testing, and elicit student and teacher feedback. Throughout each phase of the pilot testing, student and teacher feedback was evaluated in order to determine the appropriate design changes for the subsequent phases. This design model allowed for a gradual introduction and refinement of RoboBook features.

\section{Phase I.}

The primary objective for Phase I was to familiarize students with accessing and navigating RoboBooks. This initial phase allowed RoboBooks to be tested with classroom setup and technology. Additionally, the researchers collected preliminary observations and initial impressions from students and teachers pertaining to navigation $\&$ information presentation. Students were presented with curriculum congruent with course introductions, as advised by the teachers. The content was presented with various combinations of text, supplementary audio, videos, simulations, games, and activities. For all phases, content topics were selected by the teachers and the curriculum was verified by the teachers prior to classroom testing. Refinements and edits were made from their initial feedback. 
Preliminary results from the first phase indicated that students prefer to choose a method of response, as opposed to being required to answer a question with a mandatory response type. Feedback about personal progress was important for teachers and students, especially for longer tasks, to provide affirmation that the student is progressing in the right direction. Supportive media such as accompanying videos or simulations, mechanisms added to increase engagement should be pertinent and engaging, as alternatively this can result in an overall disengagement from the RoboBook environment and thus the task at hand. Also, the aesthetics of the interface should be colorful and interesting, as this was found to promote overall engagement with the interface.

\section{Phase II.}

Objectives for the second phase included providing examples of RoboBook activities that utilize UDL tools, creating activities with means for pacing, and collecting preliminary data pertaining to usability with students. The second phase was designed to provide the students with multiple response options for all answer prompts, a new color scheme, and higher fidelity videos and simulations corresponding more directly to the course material. Teacher checkpoints (incorporating group discussions within the material) were included in order to test a more integrated classroom style, as well as the addition of regular, automated assessment and feedback to the students that indicated a level of subject mastery as they progressed. Cognitive supports, such as highlighting, concept maps, and hints were also introduced in this phase.

Outcomes from the second phase indicated a need for retesting UDL tools, as many students were unaware of their addition to this phase of the design. This indicated a need for creating awareness to design changes and potential navigation considerations for initial instances of new features. Teacher checkpoints, while aiding in classroom management, felt contrived. This created a new major objective for the third phase, and resulted in trialing different classroom setups by working directly with the teachers to integrate the curriculum into the digital platform.

\section{Phase III.}

The objectives of the third phase were to integrate teacher curriculum and focus on the presenting a science concept in a learning through design context, retest UDL tools, and pilot learning outcome evaluation in the RoboBooks platform. The researchers also performed detailed observations, conducted teacher interviews, and provided refined usability surveys to the students. Several design features were included from working with the teachers and included: activity timers, multiple choice questions that provided new content representation upon an incorrect answer selection, and mathematical calculation supports created specifically for activity tasks.

As an example, on of the three-day lesson plans covered the topic of acceleration. In this lesson the students were provided different activities to guide them through understanding acceleration both through digital content within the RoboBooks environment and via a hands-on physical model. The problem state was defined by 
viewing simulations of cars in different acceleration states. The students were provided information resources to explore throughout the RoboBook in order to understand this concept. The students were additionally provided a multivariate ramp constructed from LEGO NXT kits, as seen in Figure 1. This ramp had multiple configurations that could be structured in order to learn different elements about the acceleration concept. Data was collected and analyzed within the RoboBook environment as seen in Figure 2. This data aided in understanding the problem state constraints and help lead students to making future design decisions regarding their projects. The students were lastly asked to create physical representations of acceleration through simulated movement in a stop motion animation, Figure 3. The class reviewed the movies of each group and opportunities for revised solutions were provided. Through the inquiry-based process the students constructed and revised their understanding of the physics concepts.

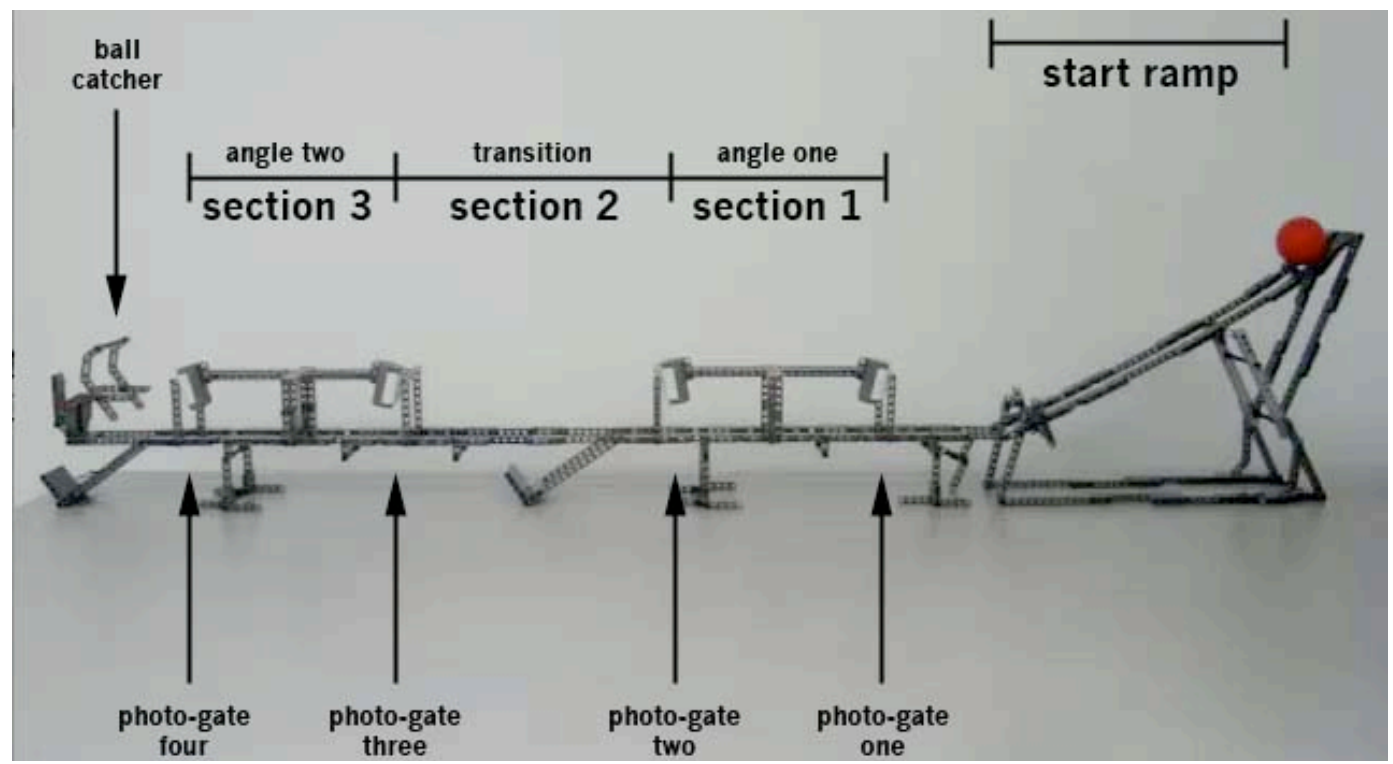

Image 1. Exploring acceleration through student modifiable ramp 


\section{R0B:B00}

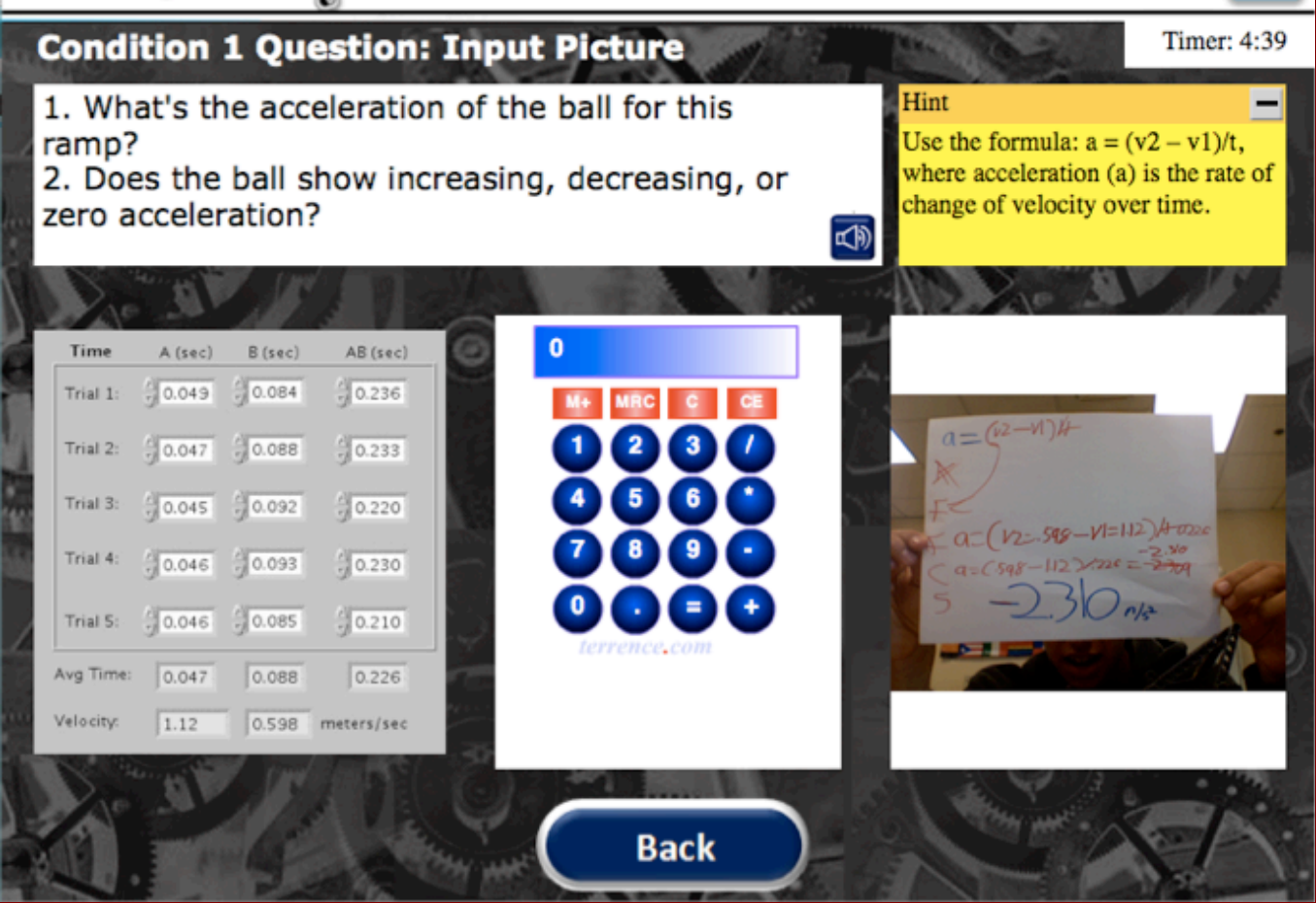

Figure 2. Explaining the criteria with ramp data within RoboBook environment

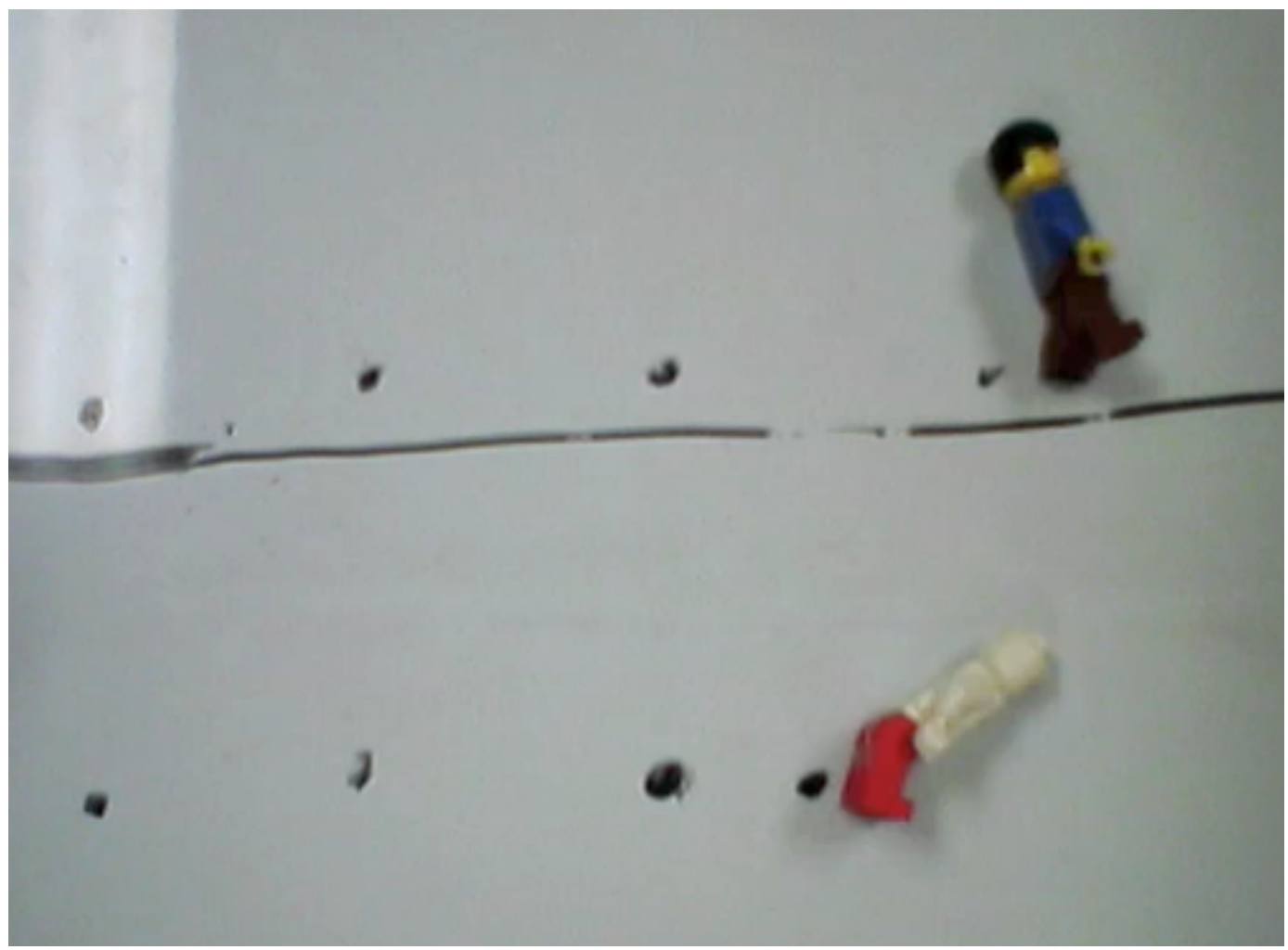

Figure 3. Student representation of acceleration problem 
Third phase outcomes included discrepancies in the utility of UDL tools with certain activity objectives. This alludes to task dependencies in UDL tool utilization, a focus for the final design phase. Classroom setup identified the need for additional teacher supports to best enable facilitating student learning. Preliminary student cognitive and usability feedback provided insight into the utility of the digital platform as a testing environment as well as the user evaluation of the software, as further described below.

\section{Cognitive Gains}

Table 1 shows preliminary pre-post data gathered from the survey items designed to explore the learning effects of participating in the RoboBook project. In questions 1-3, the students were asked to evaluate simulations of cars in motion over time in order to determine states of acceleration, deceleration, and constant velocity. Questions 3-6 were used to further probe understanding of the acceleration formula in terms of criteria and constraints. Each question was scored such that the students received a 0 for an incorrect response, a 1 for a partially correct response, and a 2 for a completely correct response. The mean and standard deviation data is from one classroom of high school students $(n=8)$. No inferential statistics were calculated for this group due to the small number of participants. All students were from the same class, had varying IEPs, and were males of ages 13-15. Thus no further disaggregation of the data was conducted.

Table 1. Learning Gains

\begin{tabular}{|l|c|c|c|c|}
\hline & Mean: Pre & Std. Deviation: Pre & Mean: Post & Std. Deviation: Post \\
\hline Question 1 & 0.25 & 0.46 & 0.83 & 0.75 \\
\hline Question 2 & 0.25 & 0.46 & 1.17 & 0.75 \\
\hline Question 3 & 0.25 & 0.46 & 0.33 & 0.52 \\
\hline Question 4 & 1.00 & 1.07 & 1.83 & 0.41 \\
\hline Question 5 & 1.00 & 1.07 & 1.00 & 1.10 \\
\hline Question 6 & 0.25 & 0.71 & 1.00 & 1.10 \\
\hline Total & $\mathbf{3 . 3 3}$ & & $\mathbf{6 . 1 7}$ & \\
\hline
\end{tabular}

This preliminary evaluation of cognitive outcomes shows pre to post test score improvement for all questions except question 5, where there was no mean change. Comparing mean values indicates an overall positive trend in performance outcomes. Though not used to characterize the student population, from a preliminary evaluative standpoint using the RoboBooks environment appears to have potential for promoting learning gains for students with disabilities. For the final phase of the design a full formative evaluation will be conducted for all students pre and post treatment in order to conclusively determine whether there was a satisfactory achievement in learning objectives by the students while using the RoboBooks software. 


\section{Usability Evaluation}

Students were surveyed upon completion of the RoboBooks activities for both high school classes. The RoboBook activities were held over 3 class periods. Responses from students who were not present for all classes were removed from the data analysis. A total of 28 students are represented in the table below, of which 17 were males and 11 females. The student ages ranged from 13-16 and represent an integrated classroom, with roughly $25 \%$ of the class being comprised of students with IEPs. The evaluation questions looks at the 22 items designed to gather information on the uses of, and responses to, various RoboBook features, tools, and resources. Each class was provided different content in the RoboBook, but all features, except for the periodic table, were provided in the RoboBooks for both classes.

Table 2 summarizes the students' responses. All items were found to be significant, meaning that the students were generally united in their responses to each of the items. There were no between schools differences aside from the preference of the calculator and understanding the science content, for which students from one class reported higher values. The 4-point Likert scale from which they selected their responses ranged from most negative (1) to most positive (4), with the intervals evenly spaced.

Table 2. Behavioral Subjective Responses

\begin{tabular}{|l|c|c|}
\hline \multicolumn{1}{|c|}{ Item } & Mean & SD \\
\hline The option to record audio for your responses? & 2.25 & 1.14 \\
\hline The option to type for your responses? & 3.39 & 0.86 \\
\hline The option to take pictures for your responses? & 2.96 & 1.14 \\
\hline The option to make a stop motion movie for your responses? & 3.32 & 0.91 \\
\hline The videos about science concepts? & 3.52 & 0.59 \\
\hline The text read out loud? & 2.79 & 1.22 \\
\hline The activity timer? & 2.11 & 1.03 \\
\hline The questions with right or wrong feedback? & 3.39 & .88 \\
\hline The teacher RoboBook projected on the whiteboard? & 3.52 & 0.79 \\
\hline The movies that went with a text passage? & 3.43 & 0.84 \\
\hline The images that went with a text passage? & 3.41 & 0.75 \\
\hline The concept map? & 2.79 & 1.26 \\
\hline The highlighting? & 2.60 & 1.20 \\
\hline The hints? & 3.25 & 1.00 \\
\hline The calculator? & 2.75 & 1.32 \\
\hline The averaging tool? & 2.96 & 1.21 \\
\hline The games and simulations? & 2.54 & 1.17 \\
\hline The science content that was presented in the RoboBook? & 3.29 & 3.10 \\
\hline Did RoboBooks help you understand the science information? & 3.11 & 0.79 \\
\hline Would you like to use RoboBooks in the future? & 3.29 & 0.71 \\
\hline Overall, did you enjoy learning about science when using & & \\
RoboBooks in class? & 3.29 & 0.66 \\
\hline Did you enjoy working on science activities in a digital environment? & 3.44 & 0.64 \\
\hline
\end{tabular}


For the user evaluation, the mean values tend toward the positive, with only 2 of the 22 items ( $9 \%$ ) below the 2.5 threshold for moving into more positive judgments. $59 \%$ of the items generated mean responses in excess of 3.0. The narrow standard deviations provide additional indication of the finding of significance for all items; the students were strongly united in their assessments of the many features of RoboBooks.

\section{Future Steps}

Several questions have emerged to pursue for the final design phase. The researchers intend to study how the content may vary utilization and preference of the RoboBook features. Utilization of features through student navigation will be correlated to self-reported feedback on preferences. Refined formative evaluation will be necessary to validate the utility of the RoboBooks tool to determine if learning objectives are being met. And lastly, a final summative evaluation will determine the overall effectiveness of the RoboBooks design and determine its applicability in use for teaching science through engineering by both the students and the teachers.

\section{Conclusions}

Conducting design driven software development, with a learner-centered focus in actual classroom testing, provides the opportunity to solicit student and teacher feedback to inform the design of educational technology. Leveraging the varied features found within the digital workbook environment, this research explored multi-phase development of software enhancements and associated science through engineering activities. Each implementation not only created direct design objectives, but also focused research objectives. Ultimately by conducting learner-centered design in the classroom the researchers were able to create design decisions to best serve student and teacher needs while simultaneously developing the educational technology. This approach allowed for the informed development of UDL tools in the RoboBooks software and aims to promote extending an engineering approach to learning science concepts in a manner accessible to all students.

Bibliography

1. National Assessment of Educational Progress, 2005. Available at http://nces.ed.gov/nationsreportcard/

2. Rose, D. H., Meyer, A., \& Hitchcock, C. (2005). The universally designed classroom: Accessible curriculum and digital technologies. Cambridge, MA: Harvard Education Press.

3. Graham, S., Harris, K., MacArthur, C., \& Schwartz, S. (1991) . Writing and Writing Instruction for Students with Learning Disabilities. Learning Disability Quarterly. 14(2), 89-114.

4. National Science Education Standards, 1996. Available at http://www.nap.edu/html/nses/ 
5. Wallace, R., Soloway, E., Krajcik, J. S., Bos, N., Hoffman, J., Hunter, H. E., Kiskis, D., Klann, E., Peters, G., Richardson, D., \& Ronen, O. (1998). Artemis: Learner-centered design of an information seeking environment for K-12 education. Human Factors in Computing Systems: CHI '98 Conference proceedings. Los Angeles, CA.

6. Phillips, R. (2005). Pedagogical Institutional and Human Factors Influencing the Widespread Adoption of Educational technology in Higher Education. Proceedings of the 22nd annual conference of the Australasian Society for Computers in Learning in Tertiary Education. Brisbane: Ascilite.

7. van Dam, A., Becker, S., Simpson, R. (2005) Next-Generation Educational Software: Why We Need It and a Research Agenda for Getting It. EDUCAUSE Review. 40 (2) 26-43.

8. Gribbons, W. (2008). Universal Accessibility and Functionally Illiterate Populations: Implications for HCI, Design, and Testing. In A. Sears \& J. Jacko (Eds.), The Human Computer Interaction Handbook (872 - 881). Mahwah, NJ: Lawrence Erlbaum Associates.

9. Rose, D. H., \& Meyer, A. (2002). Teaching every student in the digital age: Universal Design for Learning. Alexandria, VA: ASCD.

10. Soloway, E., Guzdial, M., \& Hay, K. E. (1994). Learner-Centered Design: The Challenge for HCI in the $21^{\text {st }}$ Century. Interactions $1(2), 36-48$.

11. Bruckman, A., Bandlow, A., \& Forte, A. (2008) HCI for Kids. In A. Sears \& J. Jacko (Eds.), The Human Computer Interaction Handbook (872 - 881). Mahwah, NJ: Lawrence Erlbaum Associates.

12. Danahy, E., Goswamy, A., \& Rogers, C. (2008). Future of robotics education: The design and creation of interactive notebooks for teaching robotic concepts. IEEE International Conference on Technologies for Practical Robotic Applications. Woburn, MA.

13. Kolodner, J., Camp, P., Crismond, D., Fasse, B., Gray, J., Holbrook, J., Puntambekar, S., \& Ryan, M. (2003). Problem-based learning meets case-based reasoning in the middle-school science classroom: Putting learning by design into practice. Journal of the Learning Science, 12(4), 495-547.

14. Duckworth, E., Piaget takes a teacher's look. Learning, 2(2), 1973, pp. 22-27.

15. Hynes, M., Crismond, D., Danahy, E. (2010). Using RoboBooks to teach middle school engineering and robotics. Paper presented at the American Society for Engineering Education Annual Conference \& Exposition, Louisville, $K Y$.

16. Bohman, P. \& Anderson, S. (2005) A conceptual framework for accessibility tools to benefit users with cognitive disabilities. W4A '05 Proceedings of the 2005 International Cross-Disciplinary Workshop on Web Accessibility. 85-89. 